\title{
Documento
}

\section{Percepción y disposición a donar órganos en Chile*}

\author{
Miguel Bustamante-Ubilla** \\ Patricia Villarreal-Navarrete*** \\ Carolina Cisternas-Ramírez****
}

Sumario: 1. Introducción; 2. Material y métodos; 3. Resultados; 4. Conclusiones.

Summary: 1. Introduction; 2. Material and methods; 3. Results; 4. Findings.

Palabras clave: Política pública de salud, donación de órganos y trasplantes, percepción y actitud a la donación, persona y familia en la donación de órganos.

Key WORDs: Public politics of health, organ donation and transplants, perceptions and attitudes towards donating, the individual and the family in organ donation.

Como orientación a la política pública de salud, el presente documento determina la percepción y la disposición de las personas a la donación de órganos. El instrumento de indagación fue un cuestionario aplicado a una muestra de 204 personas localizadas en 10 ciudades de Chile. La varianza del estudio fue $18,8 \%$, el error un 2,58\%, la confiabilidad, medida por Alfa de Cronbach, fue de 0,777. Las variables se identificaron mediante el método de grupo foco, se midieron por escalas nominales y ordinales. En general se concluye que tanto quienes están inscritos como donantes como aquellos que no lo están tienen una buena disposición a donar órganos y perciben que la decisión de donación debe ser tomada en forma personal, pero en conocimiento de la familia.

\footnotetext{
* Artículo recibido en abr. 2010 y aprobado en nov. 2010.

** Doctor. Dirección: Facultad de Ciencias Empresariales, Universidad de Talca, Chile. Casilla 721, Talca, Chile.E-mail: mabu@utalca.cl.

*** Dirección: Facultad de Ciencias Empresariales, Universidad de Talca, Chile. Casilla 721, Talca, Chile.

**** Dirección: Facultad de Ciencias Empresariales, Universidad de Talca, Chile. Casilla 721, Talca, Chile.
} 


\begin{abstract}
Perception and disposition to donate organs in Chile
As orientation to the public politics of health, the present document determines the perception and the disposition from people towards organ donation. The research instrument was a questionnaire applied to a sample of 204 people located in 10 cities of Chile. The variance of the study was $18,8 \%$, the error reached $2,58 \%$, the dependability, measured by Cronbach Alpha was 0,777 . The variables were identified by means of the focus group method; they were measured by nominal and ordinal scales. In general, it can be concluded that people do manifest willingness to donate organs, either if they are registered donors or if they are not. They perceive that the decision to donate should be personal, but with the knowledge of the family.
\end{abstract}

\title{
1. Introducción
}

Desde 1967, luego de la realización del primer trasplante en Chile, desde los órganos de conducción del Estado y con la participación de organizaciones privadas ocupadas de este tema, se dio inicio a un plan de estimulación y desarrollo de la donación y trasplante de órganos como mecanismo terapéutico (Mascaró, 2002). En un proceso que evidenció un progresivo incremento hasta el año 1996, pero que, sin embargo, presentó luego un lento decrecimiento (Memoria Anual 2008, 2008) que ha generado dramáticas esperas de órganos imprescindibles para lograr supervivencia (Gracia, 2001).

Datos oficiales del Estado y de organizaciones privadas muestran que, a partir del año 1998, tanto de donantes efectivos como de trasplantes realizados con donante cadáver y sobre la base de un total de 15 millones de habitantes, las tasas en millón de población (pmp) se observan sustancialmente bajas en comparación con cifras internacionales. En el año 2008 la tasa fue 7 pmp (Memoria Anual 2008, 2008) distante del óptimo que debieran situarse alrededor de $18 \mathrm{pmp}$, consecuencia de lo cual, las tasas de trasplante de órganos sólidos, de acuerdo con las patologías existentes, deberían aumentar en Chile entre 4 a 10 veces y que, según lo observado en los últimos cinco años (tabla 1), la evolución de las donaciones y trasplantes ha sido bastante desalentadora, razón por la cual indagar las percepciones y disposición de las personas resulta prioritario.

A contar del año 2009 se busca superar la tasa efectiva de $7 \mathrm{pmp}$ del 2008 calificada como la tasa más baja de la última década en Chile (Memoria Anual 2008, 2008), sin embargo, reconociendo que la percepción y disposición depende de patrones de conducta personales (Robbins, 2005), es preciso actuar conforme estos patrones. Primero, a través de actividades de procuramiento (Gracia, 2001), segundo, mediante la coordinación óptima de etapas 
clave y tercero, llegando a las fibras más profundas de la persona para generar una actitud positiva frente a la donación que redunde en un acto motivado y voluntario hacia la meta de 18 pmp (Memoria Anual 2008, 2008).

\section{Tabla 1}

Evolución de la donación de órganos en Chile

\begin{tabular}{|lrrrrrr|}
\hline & \multicolumn{7}{c|}{ Años } \\
\cline { 2 - 7 } \multicolumn{1}{c}{ Órganos } & 2004 & 2005 & 2006 & 2007 & 2008 & $2009^{*}$ \\
\hline Total Donantes & 134 & 129 & 152 & 134 & 116 & 37 \\
Riñones & 234 & 289 & 266 & 228 & 207 & 67 \\
Hígado & 67 & 62 & 87 & 75 & 72 & 19 \\
Corazón & 20 & 17 & 20 & 16 & 19 & 7 \\
Pulmón & 6 & 6 & 7 & 7 & 9 & 4 \\
Intestino & 1 & 0 & 0 & 0 & 0 & 1 \\
Páncreas & 0 & 2 & 1 & 1 & 0 & 0 \\
Injerto Válvulas Cardiacas & 65 & 53 & 47 & 20 & 4 & 5 \\
Injerto Córneas & 45 & 41 & 48 & 45 & 52 & 14 \\
Injerto Piel & 4 & 15 & 10 & 12 & 12 & 6 \\
Injerto Huesos & 3 & 10 & 7 & 7 & 9 & 4 \\
Islotes Pancreáticos & 0 & 0 & 0 & 0 & 0 & 0 \\
\hline
\end{tabular}

Fuente: Elaboración propia a partir de datos obtenidos de www.trasplante.cl.

* Meses de Enero, Febrero, Marzo y Abril del año 2009.

En lo fundamental, las actitudes corresponden a una predisposición coherente y favorable (Robbins, 2005) respecto de una situación u objeto, confirmada por la escuela de "componentes múltiples" (Deci, 1971) identificando componentes cognoscitivo, afectivo y de comportamiento. El cognoscitivo, implica saber de la existencia del objeto, así como los juicios y creencias acerca de éste; el afectivo, sintetiza los sentimientos a través del gusto - disgusto, favorable - desfavorable hacia el objeto, y el de comportamiento, refiere el actuar con alguna intención e interacción respecto de la situación u objeto (Skinner, 1977). Los determinantes centrales que inducen la conducta son activados, primero, por impulsos internos de motivación dominantes en un individuo y segundo, por estímulos del medio que influyen gradualmente con independencia de los motivos biológicos innatos, pasando a ser los dominantes en un individuo desarrollado (Ryle y otros, 1968) y que es, precisamente, lo que este trabajo investiga respecto de la donación y trasplante de órganos. 


\section{Material y métodos}

Se determinó la percepción y disposición de las personas a la donación de órganos mediante la aplicación de un cuestionario elaborado sobre la base de información secundaria de tipo cualitativo (Aaker y Day, 1996), piloteado con una submuestra de 10\% (Kinnear y Taylor, 2000) de la muestra detectándose dificultades en la comprensión de las preguntas y en la forma de responder que permitieron perfeccionar el cuestionario final. Éste incluyó escalas nominales y ordinales (Likert, 1932) medidas mediante frecuencias y por escala Thurstone de intervalos asimilable a una distribución normal (Meliá y otros, 1990) constituyendo una "distribución discriminativa" (Festinger, 1957).

La población se definió entre 15 y 64 años segmentada por edad de acuerdo con el censo del año 2002 (Instituto Nacional de Estadísticas, 2002) considerando que la edad ideal se ubica entre los 5 y 60 años (Memoria Anual 2004, 2004). Se utilizó un esquema gráfico de ubicación de las áreas habitacionales para la selección aleatoria de los encuestados (Zikmund, 1995) alcanzando una muestra total de 204 personas localizadas en 10 ciudades de Chile (tabla 2).

Tabla 2

Caracterización sociodemográfica de la muestra

\begin{tabular}{|c|c|c|c|}
\hline CONCEPTO & CARACTERÍSTICA & $\mathrm{n}$ & $\%$ \\
\hline \multirow[t]{2}{*}{ Sexo } & Femenino & 134 & 65.7 \\
\hline & Masculino & 70 & 34.3 \\
\hline \multirow[t]{5}{*}{ Intervalo de edad } & 15 a 24 años & 51 & 25.0 \\
\hline & 25 a 34 años & 45 & 22.1 \\
\hline & 35 a 44 años & 45 & 22.1 \\
\hline & 45 a 54 años & 33 & 16.2 \\
\hline & 55 a 64 años & 30 & 14.7 \\
\hline \multirow[t]{7}{*}{ Estado civil } & Casado(a) & 67 & 32.8 \\
\hline & Conviviente & 11 & 5.4 \\
\hline & Soltero(a) & 98 & 48.0 \\
\hline & Divorciado(a) & 1 & 0.5 \\
\hline & Anulado(a) & 1 & 0.5 \\
\hline & Separado(a) & 17 & 8.3 \\
\hline & Viudo(a) & 9 & 4.4 \\
\hline
\end{tabular}

Continua 


\begin{tabular}{|llcc|}
\hline CONCEPTO & \multicolumn{1}{c}{ CARACTERÍsTICA } & n & \% \\
\hline Nivel educacional & Básica incompleta & 15 & 7.4 \\
& Básica completa & 9 & 4.4 \\
& Media incompleta & 22 & 10.8 \\
& Media completa & 43 & 21.1 \\
& Superior incompleta & 68 & 33.3 \\
& Superior completa & 44 & 21.6 \\
& Postgrado & 3 & 1.5 \\
\hline
\end{tabular}

Fuente: Elaboración propia a partir de análisis descriptivos de la muestra (Programa Estadístico SPSS)

Considerando un $95 \%$ de confianza, una varianza de $18,8 \%$ calculada sobre la base de una variable dicotómica del cuestionario, el error final alcanzó a 2,58\% (Ryle y otros, 1968). La confiabilidad se verificó mediante Alfa de Cronbach (Cronbach, 1982) alcanzando a 0,777. La validez interna se verificó mediante una auditoria a los datos, la validez externa se verificó mediante la estructura estratificada de la muestra; la validez de contenido mediante la utilización del modelo de componentes múltiples (Deci, 1971), la validez de criterio se ratificó mediante la aplicación del método de grupo foco y la validez de constructo mediante consulta a expertos (Barbero, 1993) confirmándose confiabilidad y validez pertinentes (Zikmund, 1998).

\section{Resultados}

Sobre la base de una escala que va desde totalmente en desacuerdo a totalmente de acuerdo, del total de la muestra $(\mathrm{n}=204)$ un $27,9 \%$ está inscrito como donante y la diferencia no lo está (tabla 3$)$. De los inscritos $(n=57)$ un 89,5\% se manifiesta totalmente de acuerdo y de acuerdo con donar órganos y el mismo porcentaje expresa estar decididamente dispuesto a donar sus órganos. En tanto que, de los no inscritos ( $\mathrm{n}=147)$, un $69,4 \%$ está disponible para la donación de órganos y un $55,8 \%$ está totalmente de acuerdo y de acuerdo con la opción decididamente dispuesto a ser donante de órganos. De los no inscritos, un $63,2 \%$ expresa su intensión de inscribirse como donante de órganos en un futuro próximo. Finalmente, un 30,2\% de las personas consultadas optan por las opciones definitivamente no / probablemente no e indiferentes a la alternativa me inscribiría como donante de órganos. 
Tabla 3

\section{Disposición a donar órganos}

\begin{tabular}{|c|c|c|c|c|c|}
\hline & \multicolumn{4}{|c|}{ Estoy inscrito como donante de órganos } & \multirow[b]{2}{*}{$\%$} \\
\hline & & $\mathrm{SI}$ & NO & Total & \\
\hline \multirow{3}{*}{$\begin{array}{l}\text { Me gustaría ser } \\
\text { donante de órganos }\end{array}$} & Todes/Des* & 1 & 32 & 33 & 16,2 \\
\hline & Indiferente & 5 & 13 & 18 & 8,8 \\
\hline & Toac/Acu** & 51 & 102 & 153 & 75,0 \\
\hline Total & & 57 & 147 & 204 & \\
\hline \multirow[t]{3}{*}{$\%$} & & 27,9 & 72,1 & & \\
\hline & \multicolumn{4}{|c|}{ Estoy inscrito como donante de órganos } & \\
\hline & & $\mathrm{SI}$ & $\mathrm{NO}$ & Total & $\%$ \\
\hline \multirow{3}{*}{$\begin{array}{c}\text { Quiero } \\
\text { Decididamente ser } \\
\text { donante de órganos }\end{array}$} & Todes/Des & 1 & 43 & 44 & 21,6 \\
\hline & Indiferente & 5 & 22 & 27 & 13,2 \\
\hline & Toac/Acu & 51 & 82 & 133 & 65,2 \\
\hline Total & & 57 & 147 & 204 & \\
\hline \multirow[t]{3}{*}{$\%$} & & 27,9 & 72,1 & & \\
\hline & \multicolumn{4}{|c|}{ No Estoy Inscrito como donante de órganos } & \\
\hline & & $\mathrm{SI}$ & $\mathrm{NO}$ & Total & $\%$ \\
\hline \multirow{3}{*}{$\begin{array}{l}\text { Me inscribiría como } \\
\text { donante de órganos }\end{array}$} & Defno/Probnoi & & 42 & 42 & 28,6 \\
\hline & Indiferente & & 12 & 12 & 8,2 \\
\hline & Defsí /Probsíi & & 93 & 93 & 63,2 \\
\hline Total & & & 147 & 147 & \\
\hline$\%$ & & & 50 & 50 & \\
\hline
\end{tabular}

Fuente: Elaboración propia, sobre la base de datos, proveniente de la aplicación del instrumento de medición.

* Totalmente en desacuerdo / Desacuerdo i Definitivamente no / Probablemente no

** Totalmente de acuerdo / De acuerdo ii Definitivamente sí / Probablemente sí

La diferencia entre quienes quieren ser donantes de órganos y quienes quieren decididamente ser donantes de órganos alcanza a 9,8\% de las personas, por una parte, porque algunas personas aún no habían pensado en ser o no donante hasta el momento de aplicación de este cuestionario y, por otra, porque quienes están inscritos coinciden en porcentaje entre querer ser y querer decididamente ser donante de órganos, lo que no ocurre entre las personas no inscritas.

Un $13,2 \%$ se muestra indiferente y un $21,6 \%$ está totalmente en desacuerdo y en desacuerdo con la opción de donar decididamente órganos, aún cuando están inscritos. De los que no están inscritos, un $8,8 \%$ se expresa indiferente a la opción quiero decididamente ser donante de órganos.

La disposición de las personas a donar órganos se confirma mediante la prueba T-student de diferencia de medias con un T calculado igual a 53,761, 
ratificando la opción "quiere decididamente ser donante de órganos". Además, del análisis de género y con un 95\% de confianza, no se evidencia dependencia entre querer decididamente ser donante y el sexo del entrevistado.

El 52,5\% de las familias de los entrevistados declara informada de la decisión de ser o no donante de órganos (tabla 4) mientras que un 24,5\% no lo está. Se ratifica, con un $95 \%$ de confianza que de las personas que quieren decididamente ser donante de órganos $(\mathrm{n}=133$ ) un $61,6 \%$ reconoce que su familia está informada de su decisión y 18\% no lo está y que, de las familias que están informadas de la decisión, el 76,8\% está de acuerdo.

Tabla 4

Conocimiento familiar y decisión a la donación de órganos

\begin{tabular}{|c|c|c|c|c|}
\hline \multicolumn{5}{|c|}{ Mi familia está informada de mi decisión de ser (no ser) donante de órganos } \\
\hline \multicolumn{2}{|c|}{ Todes / Des * } & Indiferente & \multicolumn{2}{|c|}{ Toac / Acu ** } \\
\hline \multicolumn{2}{|l|}{50} & 47 & \multicolumn{2}{|c|}{107} \\
\hline \multicolumn{2}{|l|}{24,5} & 23 & \multicolumn{2}{|c|}{52,5} \\
\hline \multicolumn{5}{|c|}{ Mi familia está de acuerdo con mi decisión de ser (no ser) donante de órganos } \\
\hline \multicolumn{2}{|c|}{ Todes / Des } & Indiferente & \multicolumn{2}{|c|}{ Toac /Acu } \\
\hline \multicolumn{2}{|l|}{9} & 16 & \multicolumn{2}{|c|}{82} \\
\hline \multicolumn{2}{|l|}{8,4} & 15 & \multicolumn{2}{|c|}{76} \\
\hline & \multicolumn{4}{|c|}{ Mi familia está informada de mi decisión de ser donante de órganos } \\
\hline & & Todes / Des* & Indiferente & Toac / Acu** \\
\hline \multirow{3}{*}{$\begin{array}{c}\text { Quiero decididamente } \\
\text { ser donante de } \\
\text { órganos }\end{array}$} & Todes / Des & 18 & 8 & 20 \\
\hline & Indiferente & 8 & 14 & 5 \\
\hline & Toac / Acu & 24 & 27 & 82 \\
\hline & \multicolumn{4}{|c|}{ Conozco una persona trasplantada } \\
\hline \multirow{4}{*}{$\begin{array}{c}\text { Quiero decididamente } \\
\text { ser donante de } \\
\text { órganos }\end{array}$} & & & $\mathrm{SI}$ & NO \\
\hline & & / Des & 4 & 40 \\
\hline & & rente & 3 & 24 \\
\hline & & / Acu & 24 & 109 \\
\hline & \multicolumn{4}{|c|}{ Conozco una persona en lista de espera } \\
\hline \multirow{4}{*}{$\begin{array}{c}\text { Quiero decididamente } \\
\text { ser donante de } \\
\text { órganos }\end{array}$} & & & $\mathrm{SI}$ & NO \\
\hline & & / Des & 4 & 40 \\
\hline & & rente & 1 & 26 \\
\hline & & / Acu & 21 & 112 \\
\hline
\end{tabular}

